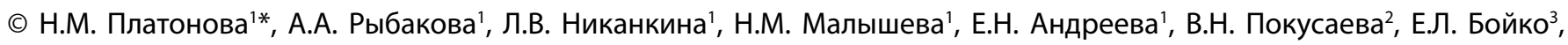
Е.А. Трошина'

'Национальный медицинский исследовательский центр эндокринологии, Москва, Россия

${ }^{2}$ Смоленский государственный медицинский университет, Смоленск, Россия

${ }^{3}$ Ивановский научно-исследовательский институт материнства и детства имени В.Н. Городкова, Иваново, Россия

ОБОСНОВАНИЕ. Известно, что витамин D играет важную роль при беременности, его недостаток может быть ассоциирован с риском развития гестационного сахарного диабета, преэклампсии, плацентарной недостаточности, низкой массой тела при рождении, может быть фактором риска преждевременных родов и бактериальных инфекций.

ЦЕЛЬ. Провести эпидемиологический анализ обеспеченности витамином D женщин на разных сроках беременности, проживающих на территории РФ.

МЕтодЫ. В наблюдательное многоцентровое поперечное сплошное исследование были включены 1198 здоровых беременных женщин, проживающих в трех регионах РФ. У всех беременных женщин определяли уровень 25(OH)D сыворотки крови, проводилось анкетирование (дата рождения и срок беременности). Сбор биоматериала проводился с августа 2018 г. по декабрь 2019 г. Конечной точкой исследования явилась оценка обеспеченности витамином D беременных женщин по триместрам. При статистическом анализе проводились подсчет и распределение по группам в зависимости от уровня витамина $\mathrm{D}$, а также подсчет медианы уровня витамина $\mathrm{D}$ в каждом регионе.

РЕЗУЛЬтАтЫ. Во всех трех исследуемых регионах подтверждено наличие дефицита витамина D у беременных женщин. Его оптимальный уровень выявлен менее чем у 7\% женщин. Недостаточность данного витамина присутствует у 20,62\% обследованных. Наибольшая доля обследованных находится в дефиците витамина D - 46,66\%. Наиболее низкие показатели выявлены в г. Смоленске, где медиана уровня витамина D составила 12,75 нг/мл. Также выявлено, что в обследованных регионах встречаемость дефицита витамина D увеличивается в динамике по триместрам.

ЗАКЛЮЧЕНИЕ. В нашем исследовании мы подтвердили наличие дефицита 25(OH)D у большинства обследованных беременных женщин, что соотносится с международными эпидемиологическими данными. Это необходимо учитывать как на этапе предгравидарной подготовки, так и для достижения оптимального уровня витамина D для каждого триместра беременности.

КЛЮЧЕВЫЕ СЛОВА: 25(ОН)D; беременность; дефицит витамина D.

\title{
VITAMIN D AND PREGNANCY: CURRENT STATE OF THE PROBLEM IN THE CENTRAL REGIONS OF THE RUSSIAN FEDERATION
}

(c) Nadezhda M. Platonova ${ }^{1 *}$, Anastasia A. Rybakova', Larisa V. Nikankina', Natalya M. Malysheva', Elena N. Andreeva', Vita N. Pokusaeva², Elena L. Boyko ${ }^{3}$, Ekaterina A. Troshina ${ }^{1}$

${ }^{1}$ Endocrinology Research Centre, Moscow, Russia

${ }^{2}$ Smolensk State Medical University, Smolensk, Russia

${ }^{3}$ Federal State Budget Institute Ivanovo scientific-research institute named after V.N. Gorodkov, Ivanovo, Russia

BACKGROUND: It has been proven that vitamin D plays an important role in pregnancy. Deficiency of this element may be associated with the risk of developing gestational diabetes mellitus, preeclampsia, placentar insufficiency, low birth weight and premature birth.

AIMS: To conduct an epidemiological analysis of vitamin D supply in women at different stages of pregnancy, living in Russia.

MATERIALS AND METHODS: We included 1198 pregnant women living in 3 regions of Russia in the observational, multicenter, cross-sectional, continuous study. All pregnant women were tested for serum 25(OH)D levels. We collected biomaterial from August 2018 to December 2019. The end point of the study was the indicator of vitamin D supply in pregnant women and its level depending on the trimester of pregnancy and region of Russia. Statistical analysis included counting and grouping according to vitamin D levels, and calculating the median vitamin D concentration in each region. 
RESULTS: We confirmed vitamin D deficiency in all regions. The optimum level of this element is present in less than $7 \%$ of women. Insufficiency of this element is present in $20,62 \%$ of all women. The largest percentage of them is in a deficit of this element - 46,66\%. We found that the lowest rates are in Smolensk, where the median was $12,75 \mathrm{ng} / \mathrm{ml}$. In addition, we found changes in vitamin D concentration: with an increase in gestational age revealed a decrease in its level.

CONCLUSIONS: In our study, we confirmed the presence of a $25(\mathrm{OH}) \mathrm{D}$ deficiency in most of the examined pregnant women, which is consistent with international epidemiological data. We should remember about these both at the stage of pre-gravid preparation and at the stage of pregnancy itself in order to achieve the optimal level of vitamin D.

KEYWORDS: Vitamin D; pregnancy; vitamin D deficiency.

\section{ВВЕДЕНИЕ}

Витамин D - жирорастворимый витамин, который присутствует в некоторых продуктах питания, может быть доступен в качестве пищевой добавки. Также он вырабатывается эндогенно при попадании ультрафиолетовых лучей на кожу.

Витамин D играет ключевую роль в регуляции фосфорно-кальциевого обмена, является эффективным средством профилактики и лечения остеомаляции и рахита, снижает риск падений у пожилых людей [1, 2].

Также современные наблюдательные исследования свидетельствуют о наличии у витамина D внескелетных эффектов, а именно его связи с аутоиммунными, сердечно-сосудистыми, пролиферативными, неврологическими заболеваниями [3, 4].

Во время беременности витамин D играет большую роль в регуляции системы мать-плацента-плод, тем самым обеспечивая нормальный рост, вес и развитие плода, снижая риски развития гипокальциемии, судорог, инфекций дыхательных путей $[5,6]$.

Анализ многочисленных исследований свидетельствует о неблагоприятном влиянии дефицита витамина D на течение и исход беременности.

С его дефицитом ассоциированы риски развития гестационного сахарного диабета, преэклампсии, плацентарной недостаточности, низкой массы тела при рождении, преждевременных родов, бактериальных инфекций [7-11]. В РФ преэклампсия встречается в 13-16\% общего числа родов [12]. Частота преждевременных родов в России составляет 5-6,5\% и колеблется в зависимости от региона [13].

Беременные и кормящие женщины относятся к группе риска по развитию дефицита и недостаточности витамина D, что подтверждается результатами многочисленных эпидемиологических исследований $[14,15]$.

Метаболизм витамина D во время беременности

Во время беременности метаболизм витамина D в организме матери претерпевает ряд физиологических изменений для обеспечения нормального роста и развития плода. Концентрация 1,25(OH), D в сыворотке крови увеличивается, начиная с ранних сроков беременности, и достигает 3-кратного повышения в III триместре. Изменение концентрации 1,25(OH) 2 D приводит к увеличению всасывания кальция в кишечнике для компенсации повышенной экскреции кальция с мочой [16].

Рост уровня 1,25(OH), D может зависеть от доступности субстрата 25(OH)D, однако механизмы данного процесса остаются не до конца изученным. Также выдвинута гипотеза о том, что ПТГ-подобные пептиды могут играть роль в регуляции фосфорно-кальциевого обмена, в частности, в увеличении концентрации $1,25(\mathrm{OH})_{2}$ D в сыворотке крови. Также на его уровень может влиять плацента, которая, как было показано в ряде экспериментальных работ, содержит фермент СYP27B1 (25-гидроксивитамина $D_{3}$ 1-альфа-гидроксилаза) и продуцирует $1,25(\mathrm{OH})_{2} \mathrm{D}$ [17].

B работе B.W. Hollis отмечено, что положительная корреляция между концентрациями 1,25(OH) 2 D и 25(OH) D более выражена у беременных женщин по сравнению с небеременными, что позволяет предположить, что синтез 1,25(OH) 2 D может зависеть от концентрации 25(OH)D в крови беременных [18].

Эпидемиология дефицита витамина D у беременных женщин

По результатам проведенных метаанализов за 19592014 гг. было показано, что средняя концентрация $25(\mathrm{OH})$ D у беременных женщин по регионам была: 18,826 нг/мл (Америка), 6-28,8 нг/мл (Европа), 5,2-24 нг/мл (Восточное Средиземноморье), 8-20,8 нг/мл (Юго-Восточная Азия), 16,8-28,8 нг/мл (Западная часть Тихого океана), 36,8 нг/мл (Африка). Исследование показало, что даже женщины, проживающие в более инсолированных регионах, находятся в дефиците или недостаточности витамина D [19]. Российские исследования показали, что беременные, проживающие в г. Санкт-Петербурге $(n=205)$, находятся в дефиците и недостаточности витамина D (17,5-22,6 нг/мл) [20]. Исследование, проведенное в Амурской области $(\mathrm{n}=60)$, выявило, что $56,7 \%$ женщин находятся в дефиците витамина D [21].

\section{ЦЕЛЬ}

Провести эпидемиологический анализ обеспеченности витамином D женщин на разных сроках беременности, проживающих на территории РФ.

\section{МАТЕРИАЛЫ И МЕТОДЫ}

\section{Дизайн исследования}

Проведено наблюдательное многоцентровое поперечное сплошное исследование, в которое были включены 1198 беременных женщин, проживающих в 3 центральных регионах РФ.

\section{Критерии соответствия}

Критерии включения: здоровые беременные женщины на разных сроках беременности в возрасте от 18 до 45 лет. 
Критерии исключения: отказ пациентки от участия в исследовании (на любом этапе), многоплодная беременность, аномалия плода, тяжелые соматические заболевания, тяжелые психические заболевания.

\section{Условия проведения и продолжительность}

исследования

Исследование проводилось на базе нескольких лечебных учреждений. Включение беременных женщин, сбор биоматериала, заполнение информированного согласия проводилось на базе женских консультаций 3 медицинских организаций: Ивановский научноисследовательский институт материнства и детства им. В.Н. Городкова, Смоленский государственный медицинский университет Министерства здравоохранения Российской Федерации, Городская клиническая больница им. В.В. Вересаева Департамента здравоохранения города Москвы. Лабораторный анализ проводился на базе Клинико-диагностической лаборатории ФГБУ «НМИЦ эндокринологии» Минздрава РФ. У каждой беременной женщины на однократном визите проводились забор крови, подписание информированного согласия, опрос.

Сбор биоматериала проводился с августа 2018 г. по декабрь 2019 г. Затем биоматериал транспортировался в ФГБУ «НМИЦ эндокринологии» Минздрава РФ, где проводились лабораторные исследования и анализ полученных результатов.

Описание медицинского вмешательства

Всем женщинам, включенным в исследование, определяли уровень 25(OH)D. Забор крови проводился рано утром, натощак. Учитывая, что наше исследование является сплошным, включение в исследование проводилось в любом триместре беременности. Кровь центрифугировали через 30-40 мин после сбора. Затем сыворотку замораживали при температуре ниже $-20^{\circ} \mathrm{C}$, с последующей подготовкой для транспортировки в ФГБУ «НМИЦ эндокринологии» Минздрава РФ. Беременные женщины заполняли анкету, где отвечали на следующие вопросы: дата рождения, срок беременности в неделях.

Уровень 25(OH)D (нг/мл) был определен методом хемилюминесцентного иммуноанализа на анализаторе Liaison XL, DiaSorin с использованием набора реактивов Liaison $25 \mathrm{OH}$ Vitamin D Total Assay. Для оценки уровня витамина D использовались клинические рекомендации Российской ассоциации эндокринологов «Дефицит витамина D у взрослых», 2016 г. (табл. 1).

Таблица 1. Оценка уровня 25(OH)D в соответствии с клиническими рекомендациями.

\begin{tabular}{lcc}
\hline \multirow{1}{*}{ Tермины } & \multicolumn{2}{c}{$\begin{array}{c}\text { Концентрация 25(OH)D } \\
\text { в сыворотке }\end{array}$} \\
\cline { 2 - 3 } & нмоль/л & нг/мл \\
\hline Выраженный дефицит & $0-25$ & $0-10$ \\
Дефицит & $25-50$ & $10-20$ \\
Недостаток & $>50-75$ & $20-30$ \\
Норма & $>75-150$ & $>30$ \\
\hline
\end{tabular}

\section{Основной исход исследования}

Конечными точками исследования были показатель обеспеченности витамином D беременных женщин, его уровень в зависимости от триместра беременности и региона проживания.

\section{Анализ в подгруппах}

Все женщины, включенные в данное исследование, были разделены по месту проживания (Москва, Иваново, (моленск), а также на 4 класса градации уровня витамина D (выраженный дефицит, дефицит, недостаточность, оптимальный уровень витамина D).

\section{Этическая экспертиза}

Данная работа была одобрена локальным этическим комитетом ФГБУ «НМИЦ эндокринологии» Минздрава России 27.09.2017, протокол №17.

\section{Статистический анализ}

Принципы расчета размера выборки. Расчет размера выборки проведен в соответствии с техническими и иными возможностями представленных медицинских организаций по сбору и хранению биоматериала и составил от 320 до 450 женщин из каждого региона. Некоторые образцы были исключены из исследования в связи с ошибками при сборе и хранении. Также исследование проведено по принципу сплошного, а не выборочного исследования, что может являться обоснованием отсутствия необходимости расчета минимального размера выборки.

Методы статистического анализа данных. Статистический анализ данных проведен с помощью программы Microsoft Excel 2016, в которой проводились подсчет и распределение по группам в зависимости от уровня витамина D, а также подсчет медианной концентрации витамина D в каждом регионе. Оценка связи триместра беременности и уровня витамина D проведена с помощью блочной диаграммы с ограничителями выбросов. Количественные показатели приведены в процентах.

\section{РЕЗУЛЬТАТЫ}

Участники исследования

В исследовании приняли участие 1198 беременных женщин, проживающих в трех центральных регионах РФ (характеристики пациенток представлены в табл. 2).

В соответствии с местом проживания женщины были разделены на 3 группы, В каждой из которых была рассчитана медианная концентрация витамина D (см. табл. 2).

Таблица 2. Распределение женщин в соответствии с регионом проживания.

\begin{tabular}{lc}
\hline Место проживания & $\begin{array}{c}\text { Медианная } \\
\text { концентрация 25(OH)D, } \\
\text { нг/мл }\end{array}$ \\
\hline Москва $(\mathrm{n}=420)$ & 16,10 \\
Иваново $(\mathrm{n}=338)$ & 16,05 \\
Смоленск $(\mathrm{n}=440)$ & 12,75 \\
\hline
\end{tabular}


Таблица 3. Распределение женщин по уровню 25(ОН)D.

\begin{tabular}{lcc}
\hline \multicolumn{1}{c}{ Градация концентрации 25(OH)D } & $\mathbf{n}$ & \% \\
\hline Выраженный дефицит (менее 10 нг/мл) & 311 & 25,96 \\
Дефицит (10-20 нг/мл) & 559 & 46,66 \\
Недостаточность (20-30 нг/мл) & 247 & 20,62 \\
Оптимальный уровень (более 30 нг/мл) & 81 & 6,76 \\
\hline
\end{tabular}

Таблица 4. Распределение по регионам.

\begin{tabular}{|c|c|c|c|c|}
\hline \multirow[t]{2}{*}{ Регионы } & \multicolumn{4}{|c|}{ Градации концентрации 25(OH)D \% } \\
\hline & $\begin{array}{c}\text { выраженный } \\
\text { дефицит } \\
\text { (менее } 10 \text { нг/мл) }\end{array}$ & $\begin{array}{c}\text { дефицит } \\
\text { (10-20 нг/мл) }\end{array}$ & $\begin{array}{c}\text { недостаточность } \\
\text { (20-30 нг/мл) }\end{array}$ & $\begin{array}{c}\text { оптимальный } \\
\text { уровень } \\
\text { (более } 30 \text { нг/мл) }\end{array}$ \\
\hline Москва $(n=420)$ & 23,1 & 42,8 & 23,3 & 10,7 \\
\hline Иваново $(n=338)$ & 21,8 & 43,4 & 26,9 & 7,6 \\
\hline Смоленск $(\mathrm{n}=440)$ & 31,8 & 52,7 & 13,1 & 2,2 \\
\hline
\end{tabular}

В соответствии с классами градации уровня витамина D женщины были разделены на 4 группы (табл. 3). Распределение по регионам в соответствии с классом градации представлено в табл. 4.

\section{Основные результаты исследования}

Во всех трех исследуемых регионах подтверждено наличие дефицита витамина D у беременных женщин. Оптимальный уровень данного показателя выявлен менее чем у 7\% женщин. Недостаточность витамина D - в 20,6\% случаев. Дефицит - у более чем 46\% обследованных женщин, выраженный дефицит - у 25\%. При анализе изменения концентрации витамина D в обследованной выборке выявлено, что концентрация 25(OH)D в III триместре была ниже, чем в I и II (рис. 1). В I триместре медиана 25(ОН)D составила 16,4 нг/мл, во II триместре - 14,9 нг/мл, в III - 13,4 нг/мл. На рисунках 2, 3, 4 представлены уровни 25(OH)D в зависимости от триместра и региона проживания.

Нежелательные явления

Нежелательные явления отмечены не были.

\section{ОБСУЖДЕНИЕ}

Исследования, проведенные с 2009 по 2013 гг., показали, что оптимальная конверсия витамина D в 1,25(OH) $D$ во время беременности достигается при уровне $25(\mathrm{OH}) \mathrm{D}$ более 40 нг/мл, и такая концентрация снижает риск преждевременных родов более чем на 50\% при сравнении с концентрацией менее 20 нг/мл и не вызывает токсических эффектов [22-25]. При этом дефицит витамина D может негативно влиять на течение беременности и развитие эмбриона уже на ранних сроках гестации.

Наше исследование является первым крупнейшим исследованием по оценке обеспеченности витамином D беременных женщин, проживающих в центральных регионах РФ. Нами была выявлена высокая распространенность дефицита витамина D у обследованных беременных женщин (46,6\%), что может быть связано с климатическими особенностями, характером питания, использованием солнцезащитных средств.

Несмотря на широкое использование в перинатальный период поливитаминных комплексов, дефицит

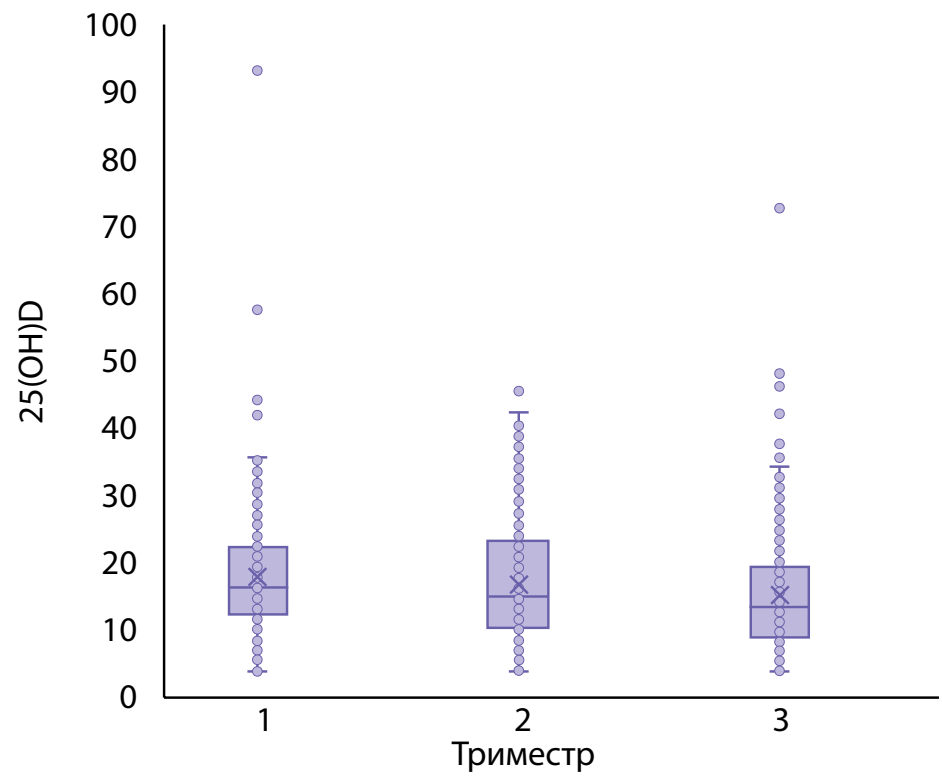

Рисунок 1. Зависимость 25(ОН)D в зависимости от триместра беременности. На оси Х представлено распределение по триместрам беременности, на оси Y - уровень 25(ОН)D (нг/мл). 


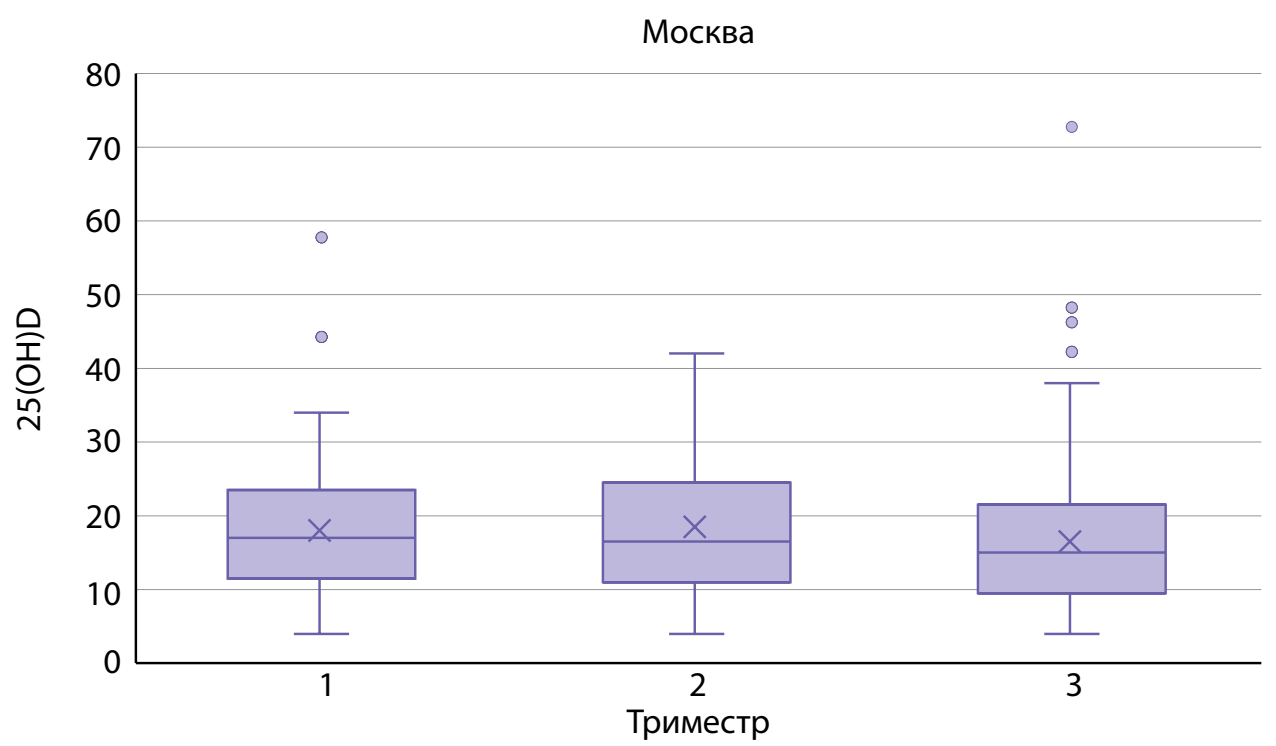

Рисунок 2. Уровень 25(OH)D в зависимости от триместра в г. Москве. На оси X представлено распределение по триместрам беременности, на оси Y - уровень 25(ОН)D (нг/мл).

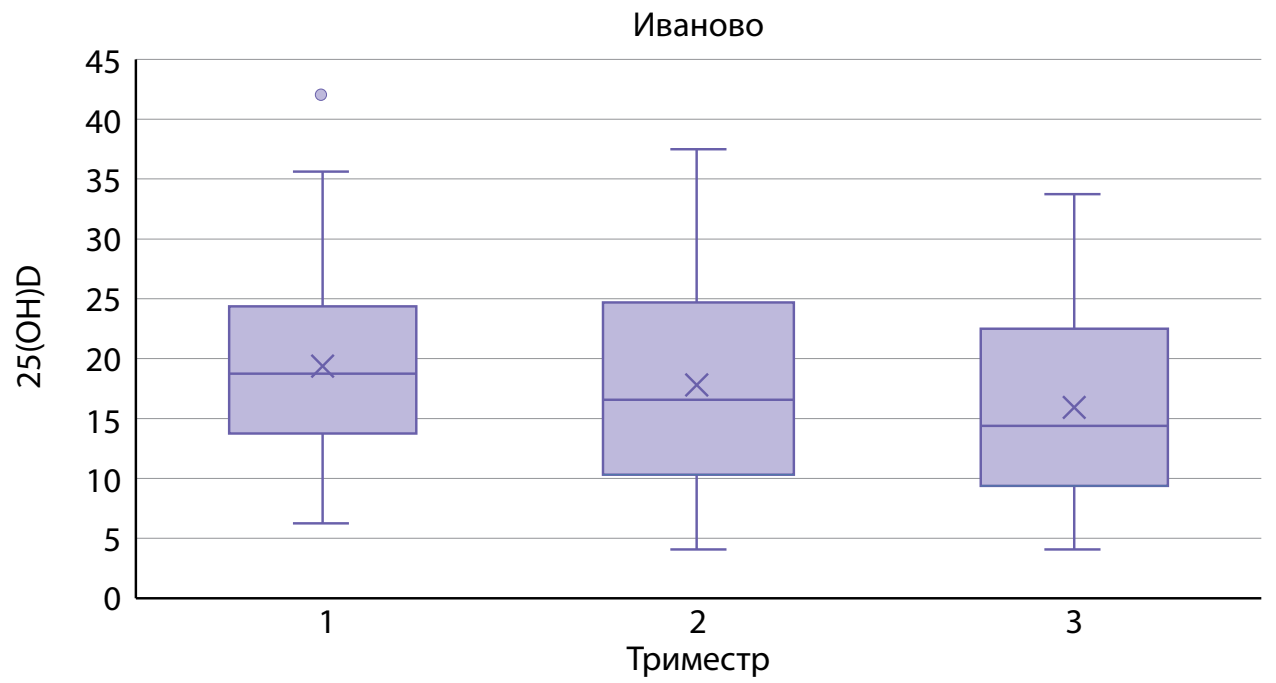

Рисунок 3. Уровень 25(ОН)D в зависимости от триместра в г. Иваново. На оси X представлено распределение по триместрам беременности, на оси Y - уровень 25(ОН)D (нг/мл).

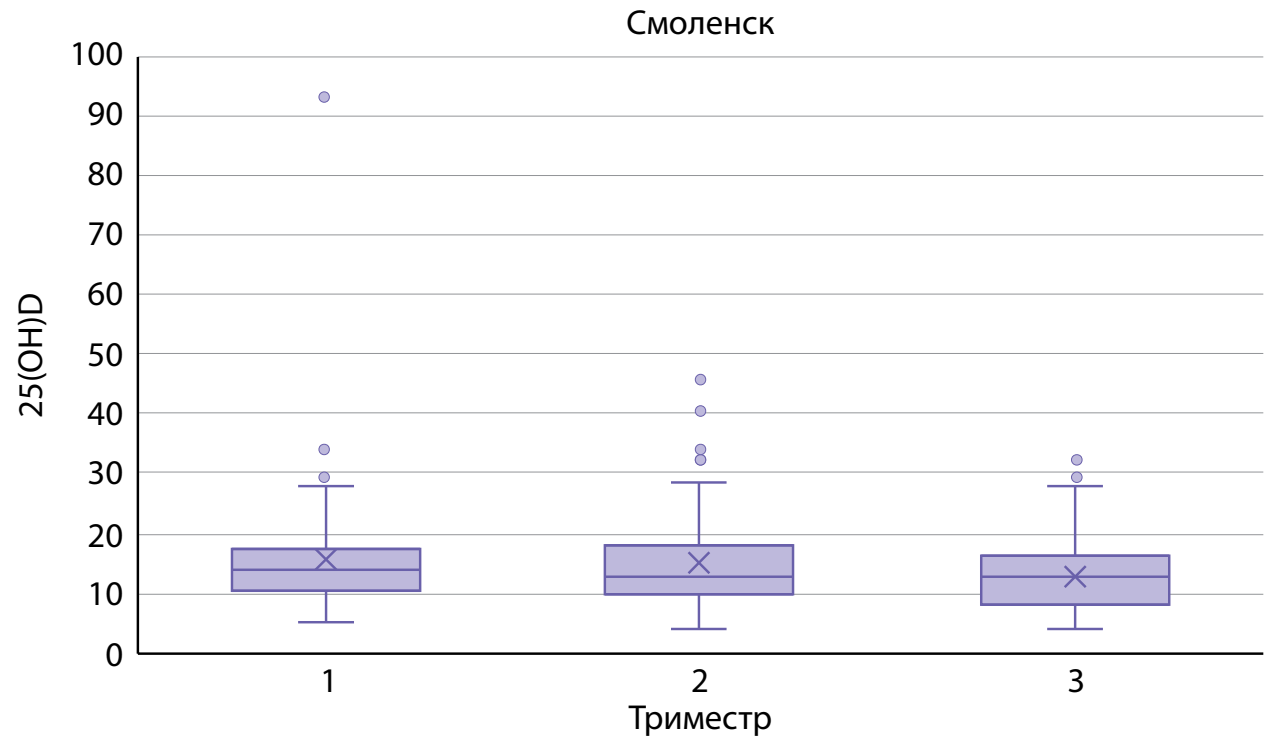

Рисунок 4. Уровень 25(ОН)D в зависимости от триместра в г. Смоленске. На оси Х представлено распределение по триместрам беременности, на оси $\mathrm{Y}-$ уровень 25(ОН)D (нг/мл). 
витамина D распространен во время беременности в связи тем, что в данных препаратах используются низкие дозы витамина D (в среднем 500 ME). Поэтому для снижения рисков осложнений, ассоциированных с дефицитом витамина $D$, еще на этапе планирования беременности необходимо достижение оптимального уровня данного показателя.

В разных странах существуют свои национальные рекомендации, касающиеся назначения витамина D с целью профилактики его дефицита. Такие рекомендации разработаны и для беременных женщин.

В клинических рекомендациях Эндокринного общества по профилактике и лечению дефицита витамина D от 2011 г. всем беременным женщинам рекомендовался прием колекальциферола 600 MЕ/сут, с верхним пределом $4000 \mathrm{ME} / с у$, а беременным женщинам, имеющим риск развития дефицита витамина $D$, - прием 1500-2000 МЕ/сут, с верхним пределом 10000 МЕ/сут [26].

В российских Клинических рекомендациях по дефициту витамина D у взрослых от 2016 г. беременным женщинам рекомендовался прием колекальциферола 800-1200 $\mathrm{ME} /$ сут без определения уровня 25(OH)D, при выявлении дефицита витамина D — прием 1500-4000 ME/сут [27, 28].

В 2020 г. в «Междисциплинарном руководстве по профилактике и лечении дефицита витамина D в прегравидарном периоде, во время беременности и после родов» сформулированы следующие рекомендации по лечению дефицита и недостаточности витамина D: женщинам, планирующим беременность (и без возможности определения уровня 25(OH)D), рекомендован прием колекальциферола 800-2000 МЕ/сут; при выявлении дефицита/недостаточности витамина D рекомендован прием лечебных доз колекальциферола до достижения целевых значений, затем прием поддерживающей дозы [29].

\section{ЗАКЛЮЧЕНИЕ}

Исследования подтверждают, что дефицит витамина D во время беременности ассоциирован со многими неблагоприятными исходами беременности.

В нашем исследовании у беременных, проживающих в центральных регионах РФ, выявлена низкая обеспеченность витамином D. Встречаемость дефицита витамина D увеличивается в динамике по триместрам. Необходимы дальнейшие исследования по оценке обеспеченности беременных витамином D в регионах, различных по климатическим данным, а также в зависимости от особенностей питания и приема препаратов витамина D.

\section{ДОПОЛНИТЕЛЬНАЯ ИНФОРМАЦИЯ}

Источник финансирования. Работа проведена при финансовой поддержке гранта РНФ №17-75-30035 «Аутоиммунные эндокринопатии с полиорганными поражениями: геномные, постгеномные и метаболомные маркеры. Генетическое прогнозирование рисков, мониторинг, ранние предикторы, персонализированная коррекция и реабилитация».

Конфликт интересов. Авторы декларируют отсутствие явных и потенциальных конфликтов интересов, связанных с публикацией настоящей статьи.

Участие авторов. Все авторы внесли значимый вклад в проведение поисково-аналитической работы и подготовку статьи, прочли и одобрили финальную версию до публикации.

Благодарности. Выражается благодарность за организацию и контроль проведения исследования: Ивановскому научно-исследовательскому институту материнства и детства им. В.Н. Городкова, Смоленскому государственному медицинскому университету Министерства здравоохранения Российской Федерации, Городской клинической больнице им. В.В. Вересаева Департамента здравоохранения города Москвы.

\section{СПИСОК ЛИТЕРАТУРЫ | REFERENCES}

1. Bischoff-Ferrari HA, Bhasin S, Manson JE. Preventing Fractures and Falls. JAMA. 2018;319(15):1552. doi: https://doi.org/10.1001/jama.2018.4023

2. Uday $S$, Högler W. Nutritional Rickets and Osteomalacia in the Twenty-first Century: Revised Concepts, Public Health, and Prevention Strategies. Curr Osteoporos Rep. 2017;15(4):293-302. doi: https://doi.org/10.1007/s11914-017-0383-y

3. Muscogiuri G, Altieri B, Annweiler C, et al. Vitamin D and chronic diseases: the current state of the art. Arch Toxicol. 2017;91(1):97-107. doi: https://doi.org/10.1007/s00204-016-1804-x

4. Bjelakovic G, Gluud LL, Nikolova D, et al. Vitamin D supplementation for prevention of mortality in adults. Cochrane Database Syst Rev. January 2014. doi: https://doi.org/10.1002/14651858.CD007470.pub3.

5. Kılıcaslan AÖ, Kutlu R, Kilinc I, Ozberk DI. The effects of vitamin $\mathrm{D}$ supplementation during pregnancy and maternal vitamin D levels on neonatal vitamin D levels and birth parameters. J Matern Neonatal Med. 2018;31(13):1727-1734 doi: https://doi.org/10.1080/14767058.2017.1326897

6. Lai S-H, Liao S-L, Tsai M-H, et al. Low cord-serum 25-hydroxyvitamin $D$ levels are associated with poor lung function performance and increased respiratory infection in infancy. Chin W-C, ed. PLoS One. 2017;12(3):e0173268. doi: https://doi.org/10.1371/journal.pone.0173268.

7. Roth DE, Leung M, Mesfin $E$, et al. Vitamin D supplementation during pregnancy: state of the evidence from a systematic review of randomised trials. BMJ. November 2017:j5237. doi: https://doi.org/10.1136/bmj.j5237

8. Bi WG, Nuyt AM, Weiler $\mathrm{H}$, et al. Association Between Vitamin D Supplementation During Pregnancy and Offspring Growth, Morbidity, and Mortality. JAMA Pediatr. 2018;172(7):635 doi: https://doi.org/10.1001/jamapediatrics.2018.0302

9. Zhou S-S, Tao Y-H, Huang K, et al. Vitamin D and risk of preterm birth: Up-to-date meta-analysis of randomized controlled trials and observational studies. J Obstet Gynaecol Res. 2017;43(2):247-256. doi: https://doi.org/10.1111/jog.13239

10. Palacios C, De-Regil LM, Lombardo LK, Peña-Rosas JP. Vitamin D supplementation during pregnancy: Updated meta-analysis on maternal outcomes. J Steroid Biochem Mol Biol. 2016;164:148-155. doi: https://doi.org/10.1016/j.jsbmb.2016.02.008

11. Pérez-López FR, Pasupuleti V, Mezones-Holguin E, et al. Effect of vitamin D supplementation during pregnancy on maternal and neonatal outcomes: a systematic review and meta-analysis of randomized controlled trials. Fertil Steril. 2015;103(5):1278-1288.e4. doi: https://doi.org/10.1016/j.fertnstert.2015.02.019

12. Сидорова И.С., Никитина Н.А. Современный взгляд на проблему преэклампсии: аргументы и факты // Акушерство и гинекология. 2013. - T. 12. — №5. - C. 10-16. [Sidorova IS, Nikitina NA. Sovremennyj vzgljad na problemu prejeklampsii: argumenty i fakty. Obstetrics and gynecology. 2013:12(5):10-16. (In Russ.)]

13. Серов В.Н., Тютюнник В.Л., Балушкина А.А. Способы терапии угрожающих преждевременных родов // Эффективная фармакотерапия. - 2013. - T. 18. - C. 20-25. [Serov VN, Tyutyunnik VL, Balushkina AA. Strategy of treatment of threatened preterm labor. Effektivnaa farmakoterapia. 2013;18:20-25. (In Russ.)]

14. Palacios C, Gonzalez L. Is vitamin D deficiency a major global public health problem? J Steroid Biochem Mol Biol. 2014;144:138-145. doi: https://doi.org/10.1016/j.jsbmb.2013.11.003

15. van der Pligt P, Willcox J, Szymlek-Gay E, et al. Associations of Maternal Vitamin D Deficiency with Pregnancy and Neonatal Complications in Developing Countries: A Systematic Review. Nutrients. 2018;10(5):640. doi: https://doi.org/10.3390/nu10050640

16. Karras SN, Wagner CL, Castracane VD. Understanding vitamin D metabolism in pregnancy: From physiology to pathophysiology and clinical outcomes. Metabolism. 2018;86:112-123. doi: https://doi.org/10.1016/j.metabol.2017.10.001 
17. Hollis $B W$, Wagner $C L$. New insights into the vitamin $\mathrm{D}$ requirements during pregnancy. Bone Res. 2017;5(1):17030. doi: https://doi.org/10.1038/boneres.2017.30

18. Weisman Y, Harell A, Edelstein $\mathrm{S}$, et al. 1a, 25-Dihydroxyvitamin D3, and 24,25-dihydroxyvitamin D3 in vitro synthesis by human decidua and placenta. Nature. 1979;281(5729):317-319. doi: https://doi.org/10.1038/281317a0

19. Saraf R, Morton SMB, Camargo CA, Grant CC. Global summary of maternal and newborn vitamin $D$ status a systematic review. Matern Child Nutr. 2016;12(4):647-668. doi: https://doi.org/10.1111/mcn.12210

20. Хазова ЕЛ., Ширинян Л.В., Зазерская И.Е., и др. Сезонные колебания уровня 25-гидроксихолекальциферола у беременных проживающих в Санкт-Петербурге // Гинекология. — 2015. T. 17. — №4. - C. 38-42. [Hazova EL, Shirinjan LV, Zazerskaja IE, et al. Sezonnye kolebanija urovnja 25-gidroksiholekal'ciferola u beremennyh, prozhivajushhih v Sankt-Peterburge. Gynecology. 2015;17(4):38-42. (in Russ.)].

21. Петрушкина А.А., Пигарова Е.А., Рожинская Л.Я. Эпидемиология дефицита витамина D в Российской Федерации // Остеопороз и остеопатии. - 2018. - T. 21. - №3. - C. 15-20. [Petrushkina AA, Pigarova EA, Rozhinskaya LY. The prevalence of vitamin D deficiency in Russian Federation. Osteoporosis and Bone Diseases. 2018;21(3):15-20. (In Russ.)]. doi: https://doi.org/10.14341/osteo10038

22. Wagner $\mathrm{CL}$, Baggerly $\mathrm{C}$, McDonnell $\mathrm{S}$, et al. Post-hoc analysis of vitamin $D$ status and reduced risk of preterm birth in two vitamin $D$ pregnancy cohorts compared with South Carolina March of Dimes 2009-2011 rates. J Steroid Biochem Mol Biol. 2016;155:245-251. doi: https://doi.org/10.1016/j.jsbmb.2015.10.022

23. Hollis BW, Johnson D, Hulsey TC, et al. Vitamin D supplementation during pregnancy: Double-blind, randomized clinical trial of safety and effectiveness. J Bone Miner Res. 2011;26(10):2341-2357. doi: https://doi.org/10.1002/jbmr.463

24. Wagner $\mathrm{CL}, \mathrm{McN}$ eil $\mathrm{R}$, Hamilton $\mathrm{SA}$, et al. A randomized trial of vitamin $\mathrm{D}$ supplementation in 2 community health center networks in
South Carolina. Am J Obstet Gynecol. 2013:208(2):137.e1-137.e13. doi: https://doi.org/10.1016/j.ajog.2012.10.888

25. Wagner $\mathrm{CL}$, McNeil RB, Johnson DD, et al. Health characteristics and outcomes of two randomized vitamin $\mathrm{D}$ supplementation trials during pregnancy: A combined analysis. J Steroid Biochem Mol Biol. 2013;136:313-320. doi: https://doi.org/10.1016/j.jsbmb.2013.01.002

26. Holick MF, Binkley NC, Bischoff-Ferrari HA, et al. Evaluation, Treatment, and Prevention of Vitamin D Deficiency: an Endocrine Society Clinical Practice Guideline. J Clin Endocrinol Metab. 2011;96(7):1911-1930. doi: https://doi.org/10.1210/jc.2011-0385

27. Дефицит витамина D у взрослых: диагностика, лечение и профилактика: клинические рекомендации. - М.; 2016. Российская ассоциация эндокринологов, ФГБУ «Эндокринологический научный центр» Минздрава России. [Deficit vitamina D u vzroslyh: diagnostika, lechenie i profilaktika: klinicheskie rekomendacii. Moscow; 2016. Rossijskaya associaciya endokrinologov, FGBU “Endokrinologicheskij nauchnyj centr" Minzdrava Rossii (In Russ.)].

28. Дефицит витамина D у взрослых: диагностика, лечение и профилактика. Учебное пособие: Клинические рекомендации Российской ассоциации эндокринологов / Под редакцией И.И. Дедова, Г.А. Мельниченко. - М.-Тверь: ООО «Издательство «Триада», 2020. - 48 c. [Deficit vitamina D u vzroslyh: diagnostika, lechenie i profilaktika. Uchebnoe posobie: Klinicheskie rekomendacii Rossijskojassocziacii endokrinologov. Pod redaktsiei II Dedova, GA Mel'nichenko. M.-Tver': OOO "Izdatel'stvo "Triada", 2020. 48 p. (In Russ.)].

29. Междисциплинарное руководство по профилактике и лечению дефицита витамина D в прегравидарном периоде, во время беременности и после родов (код по Международной классификации болезней 10-го пересмотра - Е55). - СанктПетербург: Эко-Вектор, 2020. 79 c. [Mezhdisciplinarnoe rukovodstvo po profilaktike ilecheniju deficita vitamina $\mathrm{D} v$ pregravidarnom periode, vo vremja beremennosti i posle rodov (kod po Mezhdunarodnoj klassifikacii boleznej 10-go peresmotra - E55). Sankt-Peterburg: Jeko-Vektor; 2020. 79 p. (In Russ.)].

Рукопись получена: 27.11.2020. Одобрена к публикации: 21.12.2020. Опубликована online: 30.12.2020.

\section{ИНФОРМАЦИЯ ОБ АВТОРАХ [AUTHORS INFO]}

*Платонова Надежда Михайловна, д.м.н. [Nadezhda M. Platonova, MD, ScD]; адрес: Россия, 117036, Москва, ул. Дм. Ульянова, д. 11 [address: 11 Dm. Ulyanova street, 117036 Moscow, Russia]; ORCID: https://orcid.org/0000-0001-6388-1544; eLibrary SPIN: 4053-3033; e-mail: doc-platonova@inbox.ru

Рыбакова Анастасия Андреевна [Anastasia A. Rybakova, MD]; ORCID: https://orcid.org/0000-0002-1248-9099; eLibrary SPIN: 8275-6161; e-mail: aamamykina@gmail.com

Никанкина Лариса Вячеславовна, к.м.н. [Larisa V. Nikankina, PhD]; ORCID: https://orcid.org/0000-0002-1120-8240; eLibrary SPIN: 2794-0008; e-mail: larisa.nikankina@yandex.ru

Малышева Наталья Михайловна, к.б.н. [Natalia M. Malysheva, PhD]; ORCID: https://orcid.org/0000-0001-7321-9052; eLibrary SPIN: 5793-2550; e-mail: natalya.m@list.ru

Андреева Елена Николаевна, А.М.H, профессор [Elena N. Andreeva, MD, ScD, professor]; ORCID: https://orcid.org/0000-0001-8425-0020; eLibrary SPIN: 1239-2937; e-mail: endogin@mail.ru

Покусаева Вита Николаевна, д.м.н., профессор [Vita N. Pokusaeva, MD, ScD, professor];

ORCID: https://orcid.org/0000-0003-4326-7007; eLibrary SPIN-код: 9573-6397; e-mail: vita.pokusaeva@yandex.ru

Бойко Елена Львовна, д.м.н. [Elena L. Boyko, MD, ScD]; ORCID: https://orcid.org/0000-0002-8907-4860;

eLibrary SPIN-код: 277-211; dr-boyko@mail.ru

Трошина Екатерина Анатольевна, Д.М.Н., профессор, член-корреспондент PAH [Ekaterina A. Troshina MD, ScD, professor]; ORCID: https://orcid.org/0000-0002-8520-8702; eLibrary SPIN: 8821-8990; e-mail: troshina@inbox.ru

\section{ЦИТИРОВАТЬ}

Платонова Н.М., Рыбакова А.А., Никанкина Л.В., Малышева Н.М., Андреева Е.Н., Покусаева В.Н., Бойко Е.Л., Трошина Е.А. Витамин D и беременность: современное состояние проблемы в центральных регионах РФ // Проблемы эндокринологии. — 2020. — Т.66. — №6. — С. 81-87. doi: https://doi.org/10.14341/probl12693

\section{TO CITE THIS ARTICLE:}

Platonova NM, Rybakova AA, Nikankina LV, Malysheva NM, Andreeva EN, Pokusaeva VN, Boyko EL, Troshina EA. Vitamin D and pregnancy: current state of the problem in the central regions of the Russian Federation. Problems of Endocrinology. 2020;66(6):81-87. doi: https://doi.org/10.14341/probl12693 